\title{
Aggressive pulmonary adenocarcinoma with new FGFR translocation and cMET mutation not responsive to crizotinib and nintedanib treatment: a case report
}

\author{
Martin Svaton ${ }^{1}$, Tomas Vanecek ${ }^{2}$, Petr Mukensnabl ${ }^{3}$, Jan Baxa ${ }^{4}$, Gabriela Krakorova ${ }^{1}$, Jiri Blazek ${ }^{1}$, \\ Milos Pesek ${ }^{1}$ \\ ${ }^{1}$ Department of Pneumology and Phthisiology, Charles University, Faculty of Medicine in Pilsen, Pilsen, Czech Republic; ${ }^{2}$ Bioptic laboratory, Pilsen, \\ Czech Republic; ${ }^{3}$ Department of Pathology, ${ }^{4}$ Department of Imaging Methods, Charles University, Faculty of Medicine in Pilsen, Pilsen, Czech \\ Republic \\ Correspondence to: Martin Svaton. Department of Pneumology and Phthisiology, Charles University, Faculty of Medicine in Pilsen, E. Benese 13, 305 \\ 99, Pilsen, Czech Republic. Email: svatonm@fnplzen.cz.
}

\begin{abstract}
The onset of routine use of the next generation sequencing (NGS) leads to discovery of new mutations in non-small cell lung cancer (NSCLC). In addition, comprehension of therapeutic potential of these genetic alterations in clinical practice is needed and required. Both, rare mutations and the therapeutic considerations they prompt, are dealt with in our case report describing a new fusion mutation of the fibroblast growth factor receptor (FGFR). Our case report describes a 45-year Caucasian female, nonsmoker, with the tyrosine-protein kinase Met (cMET) skip 14 mutation and a newly described fibroblast growth factor receptor-cholinergic receptor, nicotinic, alpha 6 (FGFR-CHNRA6) fusion. The tumor in this patient showed aggressive growth and was resistant to all treatment modalities administered (including combination chemotherapy with bevacizumab, pemetrexed and nintedanib), with the exception of very short efficacy of crizotinib. The patient died 5 months after diagnosis. According to the published literature, a theoretical future solution could be to administer multidimensional targeted therapy simultaneously.
\end{abstract}

Keywords: Fibroblast growth factor receptor (FGFR); cMET; crizotinib; nintedanib; case report

Submitted Aug 31, 2019. Accepted for publication Dec 12, 2019.

doi: $10.21037 /$ tcr.2019.12.85

View this article at: http://dx.doi.org/10.21037/tcr.2019.12.85

\section{Introduction}

Lung cancer remains one of the most common causes of cancer-related deaths (1). Adenocarcinoma is its most frequent type (2). Although lung cancer was previously thought of as a relatively homogenous disease, we now understand that it comprises subsets of diseases driven by distinct molecular changes (3). One of the most important genetic changes in tumors are driver mutations. Some of these mutations are predictive factors for targeted treatment that improves the prognosis of patients (1). Epidermal growth factor receptor, anaplastic lymphoma kinase, protooncogene B-Raf and proto-oncogene tyrosine-protein kinase ROS1 tyrosine kinase inhibitors (TKIs) have entered the clinical practice $(3,4)$. Although targetable oncogene products have been found in other adenocarcinoma patients, they are not yet routinely used in clinical practice or are still under development or researched $(2,3)$. In addition, new mutations and their combinations are being identified with the increasingly common use of NGS in clinical practice. This is in turn associated with questions on how to treat these unresearched genetic variations. We present a case of a patient with a combination of cMET mutation (exon 14 skip mutation) and a previously undescribed FGFR fusion (CHRNA6-FGFR1).

cMET is a tyrosine kinase, regulating cell growth and migration (5). cMET dysregulation has also been observed in NSCLC patients-especially non-smokers and womenwhere overexpression may have a negative prognostic 
effect (5). Exon 14 mutations are the most clinically significant genetic changes of cMET in NSCLC and are targetable with tyrosine kinase inhibitors (TKIs), including crizotinib. MET exon 14 encodes the region of binding site for ubiquitin ligase casitas B-lineage lymphoma (E3 CBL). Therefore, these mutations lead to loss of ubiquitinmediated degradation through negative regulation of CBL and promote oncogenesis (5).

FGFR is a family of four tyrosine kinase receptors (FGFR 1-4) (2). The FGFR gene undergoes alternative splicing that produces several isoforms that differ in epithelial and mesenchymal cells $(2,6)$. Binding of one of the 22 FGF ligands and then binding of the heparin sulfate glycosaminoglycan (HSAG) is needed for physiological FGFR activation. Consequently, FGFR undergoes dimerization and auto-phosphorylation, thereby activating downstream signaling (6). These pathways include mainly RAS/MAPK and PIK3/AKT/mTOR signaling (1). In addition, the entire process is controlled by a variety of feedback systems (6). However, there may be a number of genetic changes (amplification, mutations, translocations) in NSCLC that may result in oncogenic activity of FGFR. This leads to higher cell survival, invasiveness and proliferation (2). The association between FGFR and angiogenesis through links to the VEGF pathway has also been described (2). FGFR amplifications are the most common genetic aberrations in patients with squamous lung carcinomas but oncogenic effect of FGFR translocations has also been reported $(1,4)$. Therefore, FGFR appears to be a suitable therapeutic target and a number of inhibitors, either antibodies or TKIs, are being developed (2). Nintedanib is a multikinase inhibitor targeting FGFR, vascular endothelial growth factor (VEGF) and platelet-derived growth factor receptor (PDGFR). At present, nintedanib is used in higher treatment lines in lung adenocarcinomas together with docetaxel, mainly because of its antiangiogenic action (7).

CHNRA6, a member of nicotinic acetylcholine receptors (nAChR) gene family, is also located on chromosome 8 (8). nAChRs are a family of receptors that act as ligand-gated ion channels and are expressed in both the skeletal muscles and the central nervous system (9). Data suggest that the nAChR A6 subunit may play a pivotal role in in vivo channeling of ions in some parts of the brain and probably play a role in several brain diseases (9). nAChR could be involved in release of growth, angiogenic and neurotrophic factors in cancer cells and cancer microenvironment, including NSCLC (8). However, to the best of our knowledge, there is no information available on the role of

\section{CHNRA6 in NSCLC.}

\section{Case presentation}

A 45-year woman, non-smoker Caucasian, without any comorbidities, initially visited a pneumologist in July 2018. For two months, she suffered from unproductive cough, mild hemoptysis and fever, not responding to nonsteroidal anti-inflammatory drugs. Lung abscess pneumonia was suspected and the patient was treated with antibiotics (meropenem + metronidazole) during hospitalization. However, the chest X-ray did not improve, so a CT scan was added (Figure 1). CT showed a suspected lung tumor in the upper right lobe $(\max .8 .7 \mathrm{~cm})$ with atelectasis in the remainder of the lobe. Further, left-sided hilar and bilateral mediastinal lymphadenopathy and tiny exudate on the left were described. Further staging examinations showed two liver metastases and three brain metastases. Tumor was staged as T3N3M1b, stage IV (according $7^{\text {th }}$ edition of TNM classification). The tumor could not be uniquely verified by bronchoscopy, so a transthoracic biopsy was performed under CT control. Histologically, pulmonary poorly differentiated (Grade 3) adenocarcinoma was identified-immunohistochemistry (IHC) - TTF1, napsin A and CK7 positive; $\mathrm{p} 40$ and CK 5/6 negative. IHC tested estrogen receptors, GATA 3, CDX2, CA19-9, CD34, SMA and HMB45 were also negative. Genetic examination showed both the cMET mutation (exon 14 skip mutation) and the FGRF translocation (CHRNA6-FGFR1), other mutations within the NGS panel were negative (Figure 2).

The patient underwent stereotactic irradiation of the brain metastases (24.8.2018) and was subsequently admitted to our department. In addition, further two liver metastases and left adrenal metastasis were detected at this time (beginning of October 2018). Except for palpation sensitivity in the epigastrium, the physical finding was normal. We started analgesic treatment, transfused anemia, treated obstructive pneumonia and then administered (11.9.2019) the first cycle of a combination chemotherapy with carboplatin (AUC5) + paclitaxel $\left(200 \mathrm{mg} / \mathrm{m}^{2}\right)+$ bevacizumab $(15 \mathrm{mg} / \mathrm{kg})$.

Before the second cycle of the treatment, the patient presented with minor haemoptysis and progression of the primary tumor (Figure 1B). The liver metastases had also enlarged and carcinomatous lymphangiopathy emerged in the right middle and lower lung lobes. Newly, tumorous involvement of the lymph nodes of the retroperitoneum was evident. Immunotherapy was not available for patient with 

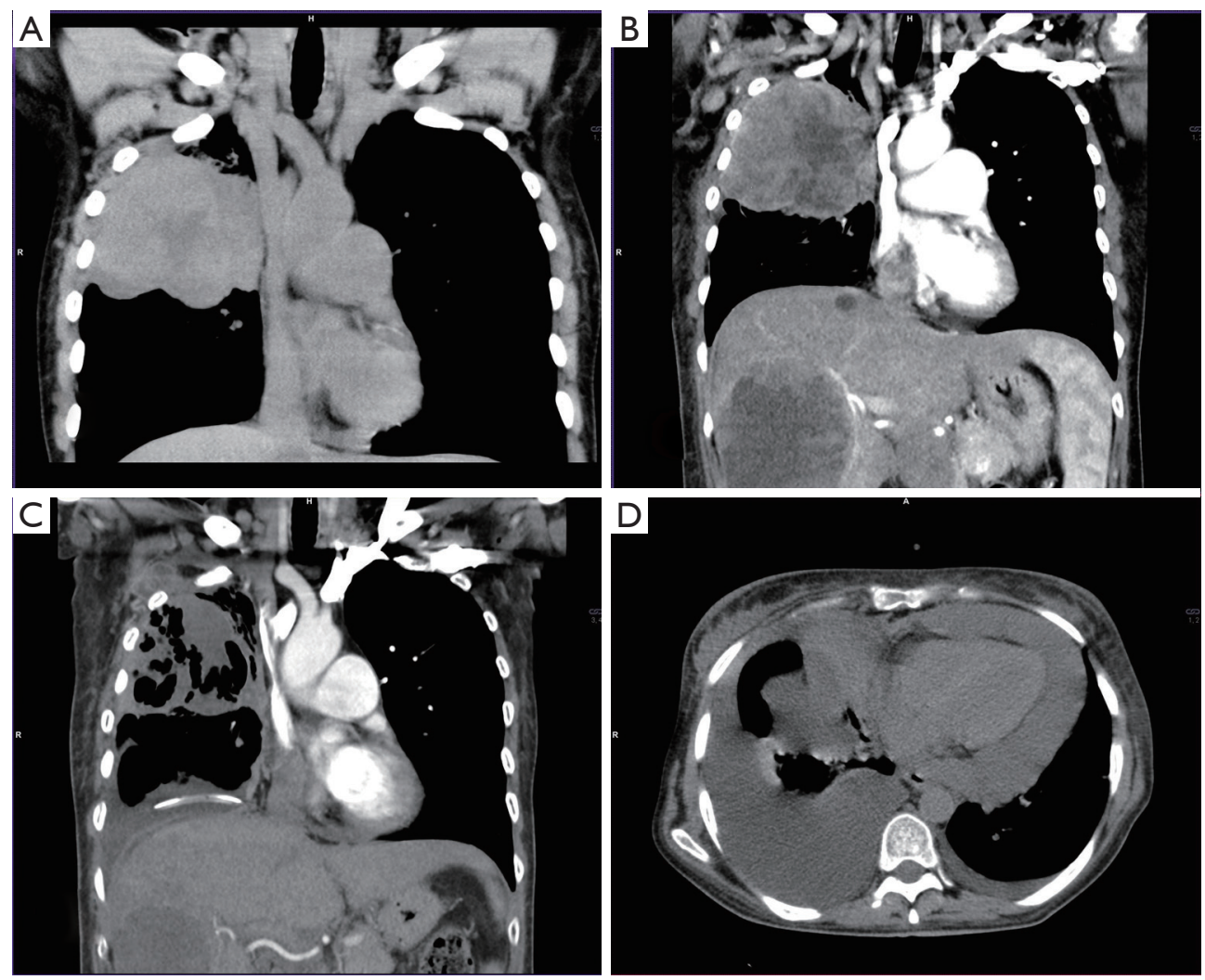

Figure 1 CT examinations showing development of tumor mass in right upper lobe. (A) Before starting chemotherapy. (B) Progressive disease after first line of therapy-new liver metastases and enlarged mediastinal lymph nodes (not showed). (C) Partial response on crizotinib therapy. (D) Progressive disease-enlargement of primary tumor, progression of malignant pleural effusion and pericardial effusion.

Eastern Cooperative Oncology Group Performance Status (ECOG PS) 2, and therefore, the second line treatment with pemetrexed $\left(500 \mathrm{mg} / \mathrm{m}^{2}\right)$ was started (10.10.2019).

The next visit showed progression of the disease with dominant left-sided malignant effusion (Figure 3). The effusion was drained and nutritional support was initiated for severe hypoalbuminemia with anasarca. Subsequently, access to crizotinib (500 $\mathrm{mg}$ BID) was secured for the patient (31.10.2019). This treatment resulted in dramatic improvement within a few days so that we were able to reduce opioid analgesic therapy. CT performed after one month of treatment showed partial regression of the primary tumor, liver metastases and lymphadenopathy (Figure 1C).

In early December 2018, we identified malignant pericardial effusion and ascites that required transient pericardial drainage. The lung scan also showed tumor progression and pneumonia. Therefore progression-free survival (PFS) of crizotinib treatment was shorter than two months (Figure 1D). After antibiotic treatment, we decided to start monotherapy (20.12.2019) with nintedanib $200 \mathrm{mg}$ BID (after agreement with the patient outside the payment of the insurance company) despite poor performance status (ECOG PS3). Unfortunately, there was no response to the treatment. The patient's condition further rapidly deteriorated and she died at the end of December 2018.

The timeline of case report is clearly shown in Figure 4.

\section{Discussion}

We report a case of a patient with novel FGFR translocation combined with cMET exon 14 skip mutation resistant to all treatments modalities administered (including combination chemotherapy with bevacizumab, pemetrexed and nintedanib) except short efficacy of crizotinib. FGFR fusions are relatively rare genetic alterations in NSCLC. 

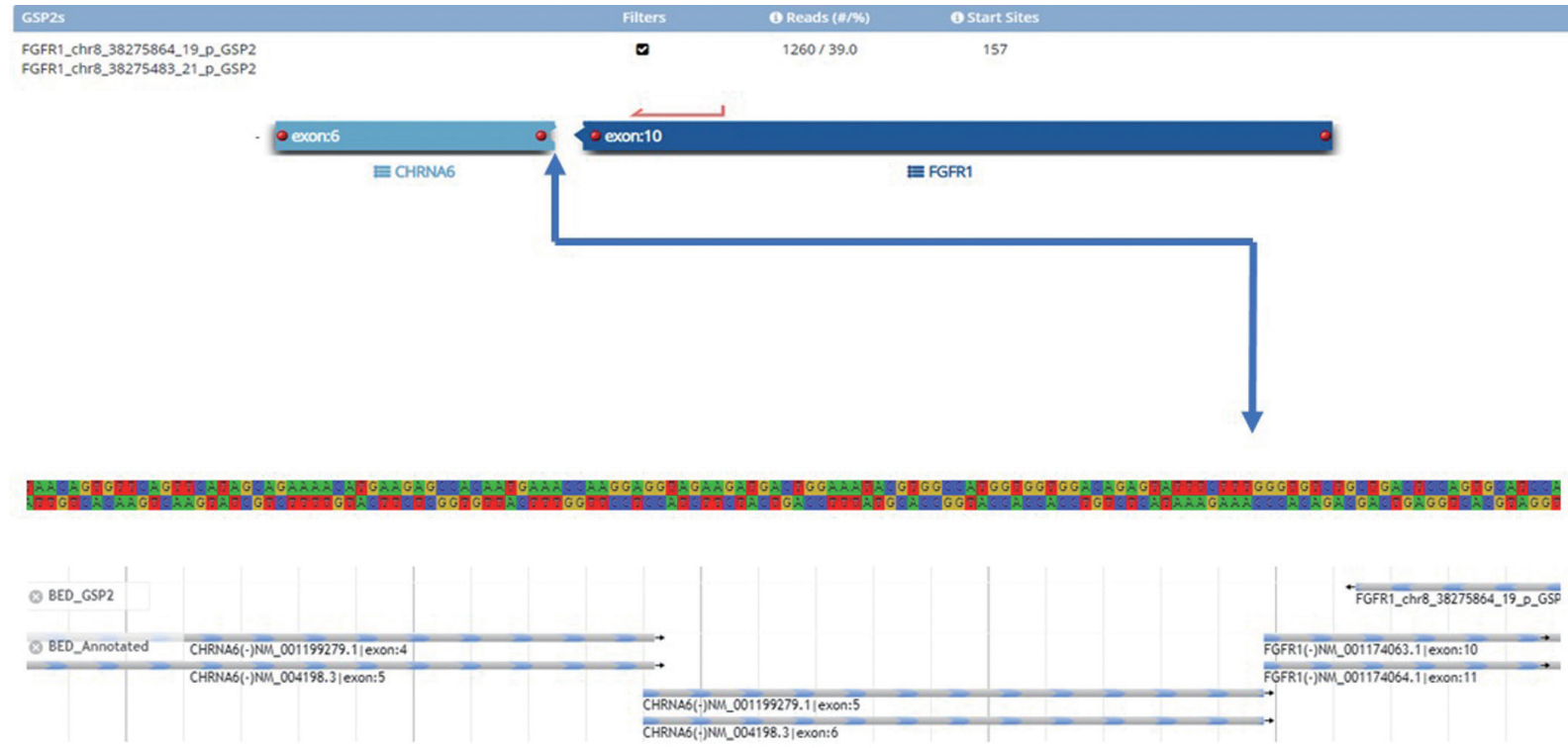

Figure 2 Detail of FGFR - CHNRA6 fusion. Fusion Plex Comprehensive Thyroid and Lung Kit (ArcherDX Inc., Boulder, CO) was used to construct cDNA library for detection gene fusions, SNV and indels in 36 genes. All steps were performed according to the manufacturer's protocol (version of the protocol LA135.F), and the library was sequenced on an Illumina platform Nextseq 500 (Illumina, San Diego, CA, USA) with at least 1,5 million reads per sample. Data were analyzed in proprietary software Archer Analysis version 5.1.7.

Wang et al. (2014) reported about $1 \%$ of NSCLC cases with FGFR translocations (4). In a large study with more than 26,000 patients, only $0.2 \%$ of NSCLC cases were positive for FGFR translocations in NGS testing (3). In both studies, FGFR translocations were more common in squamous lung cancer, although they were also reported in lung adenocarcinoma. In adenocarcinomas, FGFR translocations have more commonly been reported in non-smokers (2), as was the case in our patient. The most frequently described fusion is the FGFR3-transforming acidic coiled-coil-containing protein 3 (TACC3) but fusion partners of the FGFR1 gene have also been documented $(3,4)$. To the best of our knowledge, it is not known whether frequency of these translocations might be regionally different; our patient was not native to the Czech Republic, she lived most of her life in southern Russia.

FGFR fusions have been described in larger and less differentiated tumors, and it has been hypothesized that fusions could be associated with worse patient prognosis $(2,4)$. The poorly differentiated FGFR fusion tumor described in our case study showed very aggressive behavior that, however, could also be due to other factors, especially the influence of other genetic alterations. Although, according to the available data, FGFR fusions are rather mutually exclusive (4), parallel alterations of other oncogene drivers in at least $10 \%$ of cases have also been described (5). Similar to our case, Qin et al. (2019) described a combination of a FGFR fusion (FGFR-TACC3) with cMET mutation (3).

Sequencing seems to be the most suitable method to identify FGFR translocations, especially the NGS method, as the FISH may not detect some close fusion partners $(3,4)$. In our case, we were also able to identify close fusion partners with only the NGS and not by FISH.

Drug resistance in our patient could be due to signaling crosstalk between the individual oncogenic pathways. While negative effect of estrogen expression on FGFR has been reported in literature (9), IHC did not show estrogen expression in our patient. Resistance to antiVEGF treatment (bevacizumab used in our case) in FGFR overexpressing patients could play a more significant role $(2,10-12)$. The early cross-resistance between cMET and FGFR pathways could be also crucial (13-15). In our case, both of these genes were mutated at the same time and this could explain the early onset of resistance to crizotinib, despite the initial rapid partial regression. However, another possible cause of resistance to crizotinib cannot be excluded-e.g., the development of a resistance mutation (16). Also, we decided to use nintedanib for its effect on FGFR. However, the molecule was tested on FGFR wild type tumors and 


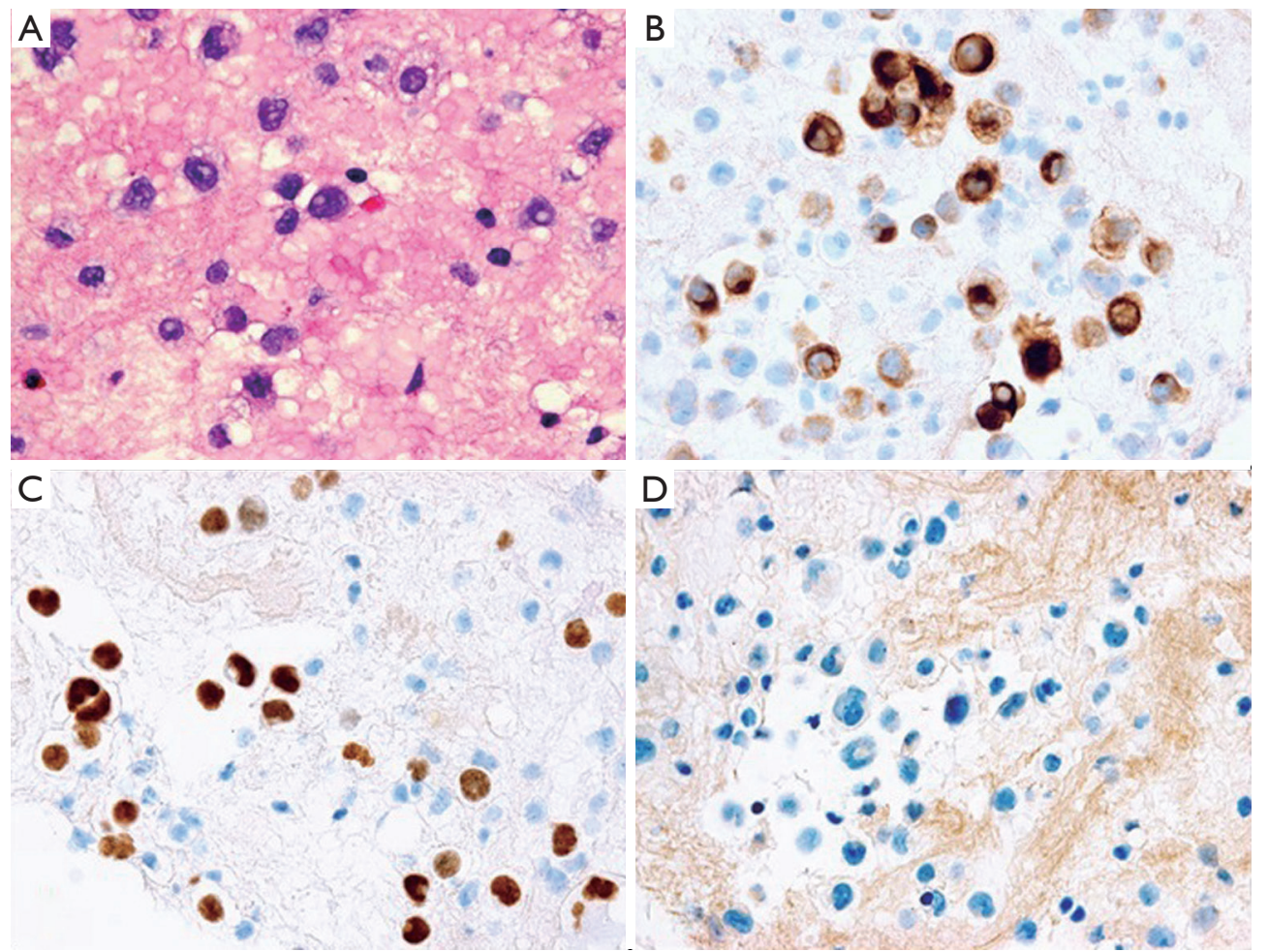

Figure 3 Cytological and immunohistochemical (IHC) examination of cytoblock from thoracic effusion. (A) Cytoblock from effusion with multiple adenocarcinoma cells with nuclear and cell polymorphism (Papanicolaou staining). (B) Cytoplasmic positivity of adenocarcinoma cells (brown color) in IHC reaction with CK7. (C) Nuclear positivity of adenocarcinoma cells (brown color) in IHC reaction with TTF1. (D) Negative reaction of adenocarcinoma cells in IHC reaction with Calretinin. Other negative IHC responses included: CK5/6, p40, D240, WT-1. The result of performed IHC reactions suggests the presence of primary lung adenocarcinoma cells and excludes mainly the possibility of malignant mesothelioma. Magnification: $200 \times$.

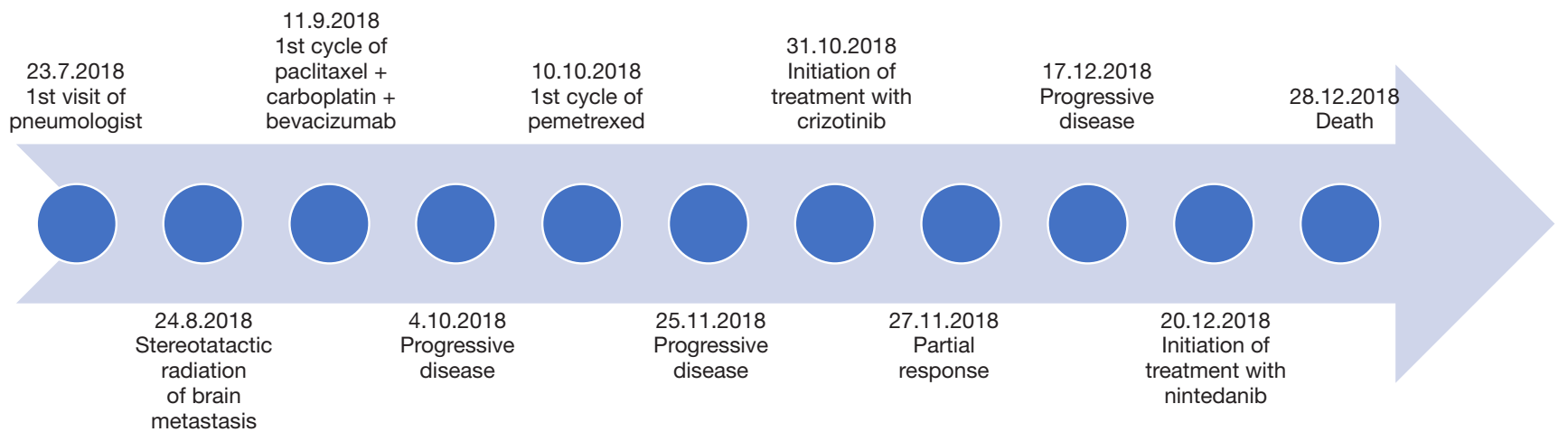

Figure 4 Case report timeline.

its efficacy in patients with FGFR alterations is not well known $(17,18)$. Hibi et al. demonstrate the possible efficacy of nintedanib in FGFR alterations in cell cultures, but data from clinical trials are lacking (7). The duration of treatment with nintedanib might also have been limited by the patient's poor PS. We also considered immunotherapy but were unable to use it since due to patient with ECOG PS2. Furthermore, data on the efficacy of immunotherapy 
in patients with FGFR alterations are lacking.

In conclusion, we report a patient with cMET skip 14 mutation and a newly described FGFR-CHNRA6 fusion. The tumor in this patient showed aggressive growth and it was resistant to all treatment modalities administered, including combination chemotherapy with bevacizumab, pemetrexed and nintedanib. Crizotinib was briefly effective with PFS of less than 2 months. Published literature suggests that simultaneously administered multidimensional targeted therapy could be the future solution for this type of patients (13).

\section{Acknowledgments}

Funding: This work was supported by AZV grant No. 1730748A.

\section{Footnote}

Conflicts of Interest: MS reports grants from Ministry of Health of the Czech Republic, during the conduct of the study; personal fees from Pharmaceutical industry - AZ, Roche, BI, BMS, MSD, outside the submitted work.

Ethical Statement: The authors are accountable for all aspects of the work in ensuring that questions related to the accuracy or integrity of any part of the work are appropriately investigated and resolved. All procedures performed in studies involving human participants were in accordance with the Declaration of Helsinki (as revised in 2013). Written informed consent was obtained from the patient (during her life) for publication of her case report and any accompanying images.

Open Access Statement: This is an Open Access article distributed in accordance with the Creative Commons Attribution-NonCommercial-NoDerivs 4.0 International License (CC BY-NC-ND 4.0), which permits the noncommercial replication and distribution of the article with the strict proviso that no changes or edits are made and the original work is properly cited (including links to both the formal publication through the relevant DOI and the license). See: https://creativecommons.org/licenses/by-nc-nd/4.0/.

\section{References}

1. Siegfried JM, Farooqui M, Rothenberger NJ, et al. Interaction between the estrogen receptor and fibroblast growth factor receptor pathways in non-small cell lung cancer. Oncotarget 2017;8:24063-76.

2. Tiseo M, Gelsomino F, Alfieri R, et al. FGFR as potential target in the treatment of squamous non small cell lung cancer. Cancer Treat Rev 2015;41:527-39.

3. Qin A, Johnson A, Ross JS, et al. Detection of Known and Novel FGFR Fusions in Non-Small Cell Lung Cancer by Comprehensive Genomic Profiling. J Thorac Oncol 2019;14:54-62.

4. Wang R, Wang L, Li Y, et al. FGFR1/3 tyrosine kinase fusions define a unique molecular subtype of non-small cell lung cancer. Clin Cancer Res 2014;20:4107-14.

5. Vuong HG, Ho ATN, Altibi AMA, et al. Clinicopathological implications of MET exon 14 mutations in non-small cell lung cancer - A systematic review and meta-analysis. Lung Cancer 2018;123:76-82.

6. Semrad TJ and Mack PC. Fibroblast growth factor signaling in non-small-cell lung cancer. Clin Lung Cancer 2012;13:90-5.

7. Hibi M, Kaneda H, Tanizaki J, et al. FGFR gene alterations in lung squamous cell carcinoma are potential targets for the multikinase inhibitor nintedanib. Cancer Sci 2016;107:1667-76.

8. Wang Y, Peng X, Zhu L, et al. Genetic variants of CHRNA5-A3 and CHRNB3-A6 predict survival of patients with advanced non-small cell lung cancer. Oncotarget 2016;7:26436-43.

9. Ebihara M, Ohba H, Ohno SI, et al. Genomic organization and promoter analysis of the human nicotinic acetylcholine receptor alpha6 subunit (CHNRA6) gene: $\mathrm{Alu}$ and other elements direct transcriptional repression. Gene 2002;298:101-8.

10. Guddo F, Fontanini G, Reina C, et al. The expression of basic fibroblast growth factor (bFGF) in tumorassociated stromal cells and vessels is inversely correlated with non-small cell lung cancer progression. Hum Pathol 1999;30:788-94.

11. Donnem T, Al-Shibli K, Al-Saad S, et al. Prognostic impact of fibroblast growth factor 2 in non-small cell lung cancer: coexpression with VEGFR-3 and PDGF-B predicts poor survival. J Thorac Oncol 2009;4:578-85.

12. Rak J, Filmus J, Kerbel RS. Reciprocal paracrine interactions between tumour cells and endothelial cells: the 'angiogenesis progression' hypothesis. Eur J Cancer 1996;32A:2438-50.

13. Kim B, Wang S, Lee JM, et al. Synthetic lethal screening reveals FGFR as one of the combinatorial targets to overcome resistance to Met-targeted therapy. Oncogene 
2015;34:1083-93.

14. Kim SM, Kim H, Yun MR, et al. Activation of the Met kinase confers acquired drug resistance in FGFR-targeted lung cancer therapy. Oncogenesis 2016;5:e241.

15. Beau-Faller M, Gaub MP, Schneider A, et al. Allelic imbalance at loci containing FGFR, FGF, c-Met and HGF candidate genes in non-small cell lung cancer sub-types, implication for progression. Eur J Cancer 2003;39:2538-47.

16. Reungwetwattana $\mathrm{T}$, Liang $\mathrm{Y}$, Zhu $\mathrm{V}$, et al. The race to target MET exon 14 skipping alterations in non-small cell lung cancer: The Why, the How, the Who, the Unknown, and the Inevitable. Lung Cancer 2017;103:27-37.

17. Hilberg F, Tontsch-Grunt U, Baum A, et al. Triple Angiokinase Inhibitor Nintedanib Directly Inhibits Tumor Cell Growth and Induces Tumor Shrinkage via Blocking Oncogenic Receptor Tyrosine Kinases. J Pharmacol Exp Ther 2018;364:494-503.

18. Bronte G, Passiglia F, Galvano A, et al. Nintedanib in NSCLC: evidence to date and place in therapy. Ther Adv Med Oncol 2016;8:188-97.

Cite this article as: Svaton $M$, Vanecek T, Mukensnabl P, Baxa J, Krakorova G, Blazek J, Pesek M. Aggressive pulmonary adenocarcinoma with new FGFR translocation and cMET mutation not responsive to crizotinib and nintedanib treatment: a case report. Transl Cancer Res 2020;9(3):2082-2088. doi: 10.21037/tcr.2019.12.85 